\title{
Modeling of Spontaneous Raman Scattering in silica light guides for Distributed Temperature Sensing
}

\author{
by I. S. M. Shatarah, R. Olbrycht, B. Więcek
}

Lodz University of Technology, Institute of Electronics, 211/215 Wólczańska St. 90-924 Lodz, Poland, iyad.shatarah@gmail.com

\begin{abstract}
Silica optical fibers are used as sensors in the Distributed Temperature Sensing (DTS) systems today. They are durable and immune to electromagnetic interferences, and it results in their reliability and the potential of application in rough industrial environments. The temperature profile along the fiber is obtained by applying the Optical Time Domain Reflectometry $(O T D R)$. There is alternative method using Optical Frequency Domain Reflectometry (OFDR). Both methods allow estimating the temperature and the distance of hot spots. This paper presents simulation models of OTDR and OFDR for the single-ended DTS system with pulse and continuous laser light generation.
\end{abstract}

\section{Introduction}

Raman spectroscopy became more and more useful in many applications today. It can be applied in the measurement systems of strain, leak, structure and temperature changes [1]. DTS systems use the spontaneous Raman scattering [2]. The Raman anti-Stokes radiation component generated in a spontaneous manner is sensitive to temperature changes. In order to obtain temperature readings, the different demodulation algorithms can be applied focusing on the ratio of anti-Stokes, Stokes or Rayleigh components [3]. In order to measure temperature and position of hot spots, DTS systems use either the Optical Time Domain or the Optical Frequency Domain Reflectometry (OFDR) $[4,5]$. DTS system configuration depends on application and environment, the system is applied for.

In this paper, two simulation models of DTS systems operating with pulse and continuous lasers are presented. The models evaluate the distribution of power along the light guide both for Stokes and anti-Stokes components of the scattered radiation. The simulations are performed for the single-ended DTS system using time and frequency domain reflectometry.

\section{Modeling of continuous and pulsed lasers DTS system}

The Bose - Einstein statistical probability distribution of energy levels of molecules is used in the analysis of the Raman Stokes and anti-Stokes scattering [5]. These statistics depend on temperature and indirectly they are functions of distance $z$ - eq. (1) and (2). Due to the inertia of heat transfer, especially in low conductive glass optical fibers, one can estimate the position of a hot spot precisely [6].

$$
\begin{gathered}
\rho_{S}(z)=\frac{1}{1-e^{-\frac{\Delta E}{k T(z)}}} \\
\rho_{A S}(z)=\frac{e^{-\frac{\Delta E}{k T(z)}}}{1-e^{-\frac{\Delta E}{k T(z)}}} \\
\Delta E=h \Delta v
\end{gathered}
$$

where: $k$ - Boltzmann constant, $h$ - Planck constant, $T(z)$ - temperature along the fiber in Kelvin, $\Delta v$ - Raman frequency shift. $\Delta E$ is the energy difference, and is about $50 \mathrm{meV}$ for vitreous $\mathrm{SiO}_{2}$.

Let's assume the DTS based on OFDR. The optical fiber has the length $L$ and light of a pumping laser is periodically modulated at different frequencies in the range $f_{\min }<f<f_{\max }-$ fig. 1 . 


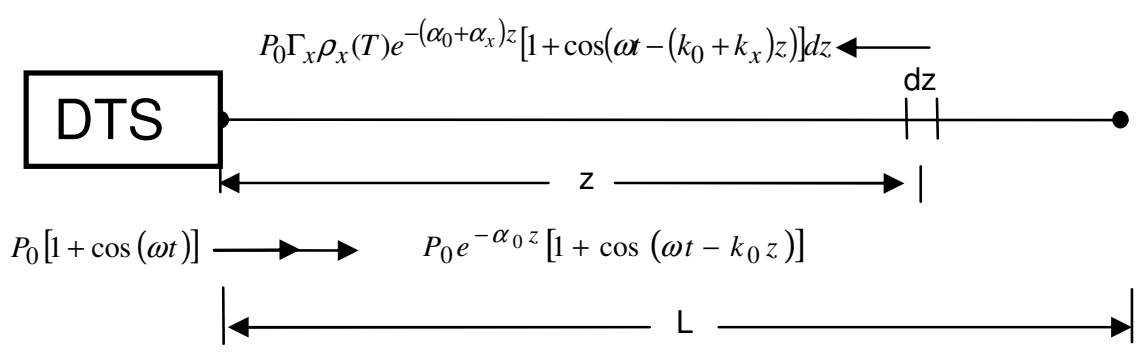

Fig. 1:Single-ended DTS system using continuously operating laser modulated with low frequency signal at $f=2 \pi / \omega$

The input power generated by the laser is $P_{0}[1+\cos (\omega t)]$. It is modulated by a low frequency signal, where angular frequency $\omega=2 \pi t$. The power of the scattered light for either Stokes or anti-Stokes components originated by $d z$ part of an optical fiber at distance $z$ from the light source can be expressed as:

$$
d P_{x}=P_{0} \Gamma_{x} \rho_{x} e^{-\alpha z}[1+\cos (\omega t-k z)] d z
$$

where: $x$ denotes Stokes (S) or anti-Stokes (AS) components of the backscattered light, attenuation coefficient $\alpha=\alpha_{0}+$ $\alpha_{x}$ consists of attenuation of pumping and scattered light, coefficient $k=k_{0}+k_{x}$ denotes a phase shift (for pumping and scattered light) of modulating signal due to the propagation of light through an optical fiber, $k=\omega n / c$.

In order to calculate the total power at the receiving point, the integration of the eq. (4) is required. It is convenient to rewrite the eq. (4) using the complex phasor notation. In addition, let's neglect the $D C$ part in the eq. (4) and consider the $A C$ component only.

$$
d P_{x}=P_{0} \Gamma_{x} \rho_{x} \operatorname{Re}\left\{e^{-(\alpha+j k) z} e^{j \omega t}\right\} d z
$$

The pumping light is back-scattering along the whole path of an optical fiber. In consequence, the detector is receiving the Stokes and anti-Stokes back-scattered radiation with different phase shifts depending on the position of scattering $(0<z<L)$. Hence, the integration over the light guide length is needed. Finally, the backscattered return power can be estimated as:

$$
P_{x}(\omega)=P_{0} \Gamma_{x} \operatorname{Re}\left\{\int_{0}^{L} \rho_{x}(z) e^{-(\alpha+j k) z} d z e^{j \omega t}\right\}
$$

As one can notice, the term under the integral symbol in the eq. (6) is the spatial Fourier Transform of the function $f_{x}(z)$ in the distance domain.

$$
f_{x}(z)=\rho_{x}(z) e^{-(\alpha+j k) z}
$$

By measuring the optical backscattered power for different modulation frequencies and applying the Inverse Fourier Transform, one can get the function $f_{x}(z)$, which directly corresponds to the temperature distribution in an optical fiber.

For the constant temperature of the whole fiber, eg.: $T=300 \mathrm{~K}$, the integral (6) can be analytically found. It has already been discussed in the literature [7]. The modulus of back-scattered power for modulation frequency $f=\omega / 2 \pi$ can be presented as:

$$
\left|P_{x}(\omega)\right|=P_{0} \Gamma_{x} \rho_{x} \sqrt{\frac{e^{-\alpha L}}{\alpha^{2}+k^{2}}[\cosh (\alpha L)-\cos (k L)]}
$$

By applying the classical signal theory, one can easily estimate the DTS system performance. The maximum length of an optical fiber is limited by the modulating frequency resolution $\Delta f$. 


$$
L_{\max }=\frac{c}{2 n \Delta f}
$$

Similarly, the spatial resolution $d z$ of DTS systems depends on maximum modulating frequency $f_{\max }$.

$$
d z=\frac{c}{2 n f_{\max }}
$$

In the DTS system based on pulsed laser and OTDR, the response time and distance from the light source is used in order to evaluate the Raman Stokes and anti-Stokes backscattering radiation power depending on temperature changes. The backscattering occurs for the distance $L$, depending on the pulse duration and the refractive index of the fiber core. Raman Stokes and anti-Stokes power takes the form of eq. (11) and (12).

$$
\begin{gathered}
P_{S}(z)=P_{0} \rho_{S}(z) \Gamma_{S} \Delta z e^{-\left(\alpha_{P 0}+\alpha_{P S}\right) z} \\
P_{A S}(z)=P_{0} \rho_{A S}(z) \Gamma_{A S} \Delta z e^{-\left(\alpha_{P 0}+\alpha_{P A S}\right) z} \\
\Delta z=\frac{\Delta t c}{n_{g r}}
\end{gathered}
$$

where: $P_{0}$ - pump power, $\Gamma_{\mathrm{s}}, \Gamma_{\text {as }}$ - Raman Stokes and anti-Stokes capture coefficients, $\alpha_{P 0}, \alpha_{P S}, \alpha_{P A S}$ - effective power attenuation coefficient for source wave, Stokes and anti-Stokes respectively, $z-$ test point distance, $\Delta t$ - pulse duration, $c$ - speed of light, $n_{g r}$ - group refractive index of the fiber's material.

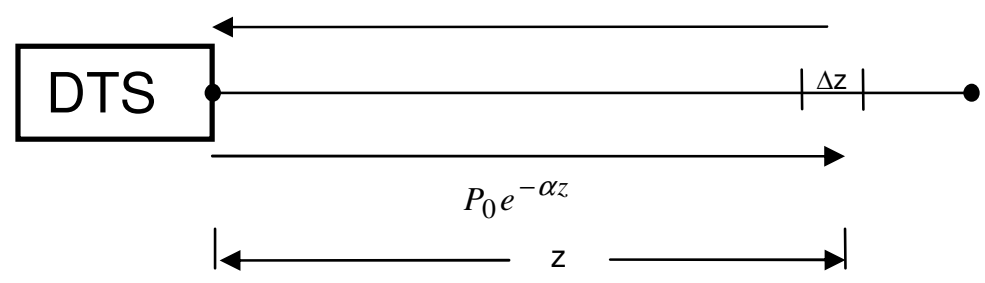

Fig. 2: Single-ended DTS system operation scheme for a pulsed laser

In order to obtain the temperature along a fiber, the demodulation algorithm is used, basing on the ratio of Raman anti-Stokes and Stokes, or Raman anti-Stokes and Rayleigh backscattered radiation intensities. The ratio between the anti-Stokes and Stokes intensities can be obtained by eq. (14) [8]:

$$
R(z, T)=\left(\frac{\lambda_{S}}{\lambda_{A S}}\right)^{4} e^{-\frac{\Delta E}{k T(z)}\left(\alpha_{P A S}-\alpha_{P S}\right) z}
$$

while the ratio of the anti-Stokes and Rayleigh radiation intensities can be obtained by [8]:

$$
R(z, T)=\left(\frac{\lambda_{P}}{\lambda_{A S}}\right)^{4} e^{-\frac{\Delta E}{k T(z)}\left(\alpha_{P A S}-\alpha_{P 0}\right) z}
$$

where: $\lambda_{P}, \lambda_{S}, \lambda_{A S}-$ Stokes and anti-Stokes wavelengths respectively, $k$ - Boltzmann constant, $T(z)$ - temperature along the fiber in Kelvin, $\alpha_{P O}, \alpha_{P S}, \alpha_{P A S}-$ effective power attenuation coefficient for Stokes and anti-Stokes respectively, $z-$ distance along the fiber. 


\section{Parameters of the models}

DTS system modeling based on Raman scattering was performed in MATLAB program environment. The assumptions of this model were chosen basing on parameters of the typical DTS system. Later, in the next phase of the study, it is planned to apply these assumptions in the development of the laboratory demo DTS system. The system, which is now under construction, uses a single-ended configuration with $5 \mathrm{~mW} / 1550 \mathrm{~nm}$ laser diode. In order to compare the achieved results, another two cases were simulated: for $850 \mathrm{~nm}$ and $1320 \mathrm{~nm}$ wavelength and the same $5 \mathrm{~mW}$ power of the laser [1]. The graded-index multimode fiber is used in this system. The pulse duration is set to $10 \mathrm{~ns}$. The values of chosen parameters necessary for DTS modeling were gathered, as shown in table 1 [5].

Table 1.Effective power attenuation coefficients and Raman Stokes and anti-Stokes capture coefficients for different wavelengths used in DTS modelling (values for a graded-index fiber) [5]

\begin{tabular}{|c|c|c|c|c|c|c|c|}
\hline \multicolumn{3}{|c|}{ Wavelength } & \multicolumn{3}{c|}{$\begin{array}{c}\text { Effective power attenuation } \\
\text { coefficients }\end{array}$} & \multicolumn{2}{c|}{$\begin{array}{c}\text { Raman Stokes and anti-Stokes capture } \\
\text { coefficients }\end{array}$} \\
\hline $\begin{array}{c}\lambda_{0} \\
\mathrm{~nm}\end{array}$ & $\begin{array}{c}\lambda_{S} \\
\mathrm{~nm}\end{array}$ & $\begin{array}{c}\lambda_{A S} \\
\mathrm{~nm}\end{array}$ & $\begin{array}{c}\alpha_{P O} \\
\mathrm{~dB} / \mathrm{km}\end{array}$ & $\begin{array}{c}\alpha_{P S} \\
\mathrm{~dB} / \mathrm{km}\end{array}$ & $\begin{array}{c}\alpha_{P A S} \\
\mathrm{~dB} / \mathrm{km}\end{array}$ & $\begin{array}{c}\Gamma_{\mathrm{S}} \\
\mathrm{m}^{-1}\end{array}$ & $\Gamma_{\mathrm{AS}}$ \\
$\mathrm{m}^{-1}$ & $118^{*} 10^{-10}$ \\
\hline 840 & 872 & 810 & 2.4 & 2 & 3 & $87.7^{*} 10^{-10}$ & $20.9^{*} 10^{-10}$ \\
\hline 1320 & 1401 & 1248 & 0.4 & 0.9 & 0.6 & $13.2^{*} 10^{-10}$ & $11.4^{*} 10^{-10}$ \\
\hline 1550 & 1663 & 1451 & 0.35 & 0.37 & 0.5 & $6.63^{*} 10^{-10}$ & $1^{-10}$ \\
\hline
\end{tabular}

One must notice the difference in the measurements units, which must be equivalent for all the variables in order to obtain correct modeling results. In this DTS modeling, it is assumed that the length of the multimode fiber is $1000 \mathrm{~m}$, and that the temperature along the fiber is constant $\left(27^{\circ} \mathrm{C}\right)$ except for a chosen section, where the temperature is higher $\left(47^{\circ} \mathrm{C}\right)$, thus a peak can be observed in the response of the Stokes and anti-Stokes radiation lines in the case of temperature changing. The ratio between the anti-Stokes and Stokes intensities was also calculated.

\section{Results}

Figures 3-8 present the modeling results of DTS system for three cases of modulated, continuously operating laser diodes at the wavelength $850 \mathrm{~nm}, 1320 \mathrm{~nm}$ and $1550 \mathrm{~nm}$. The level of Stokes and anti-Stokes power decreases with the increasing wavelength of the laser diodes. The frequency range of the modulating signal of $10 \mathrm{kHz}-100 \mathrm{MHz}$ allows obtaining the spatial resolution of $d z \approx 1 \mathrm{~m}$. The maximum length of the fiber is $L_{\max } \approx 10 \mathrm{~km}$, for the system with frequency step $d f=10 \mathrm{kHz}$. The results of Inverse Fourier Transform show that the higher the wavelength, the higher the power levels of the received back-scattered radiation. Anti-Stokes lines are much more sensitive to temperature changes, thus their peaks at higher temperature are higher than the Stokes lines for each wavelength of the laser light. 


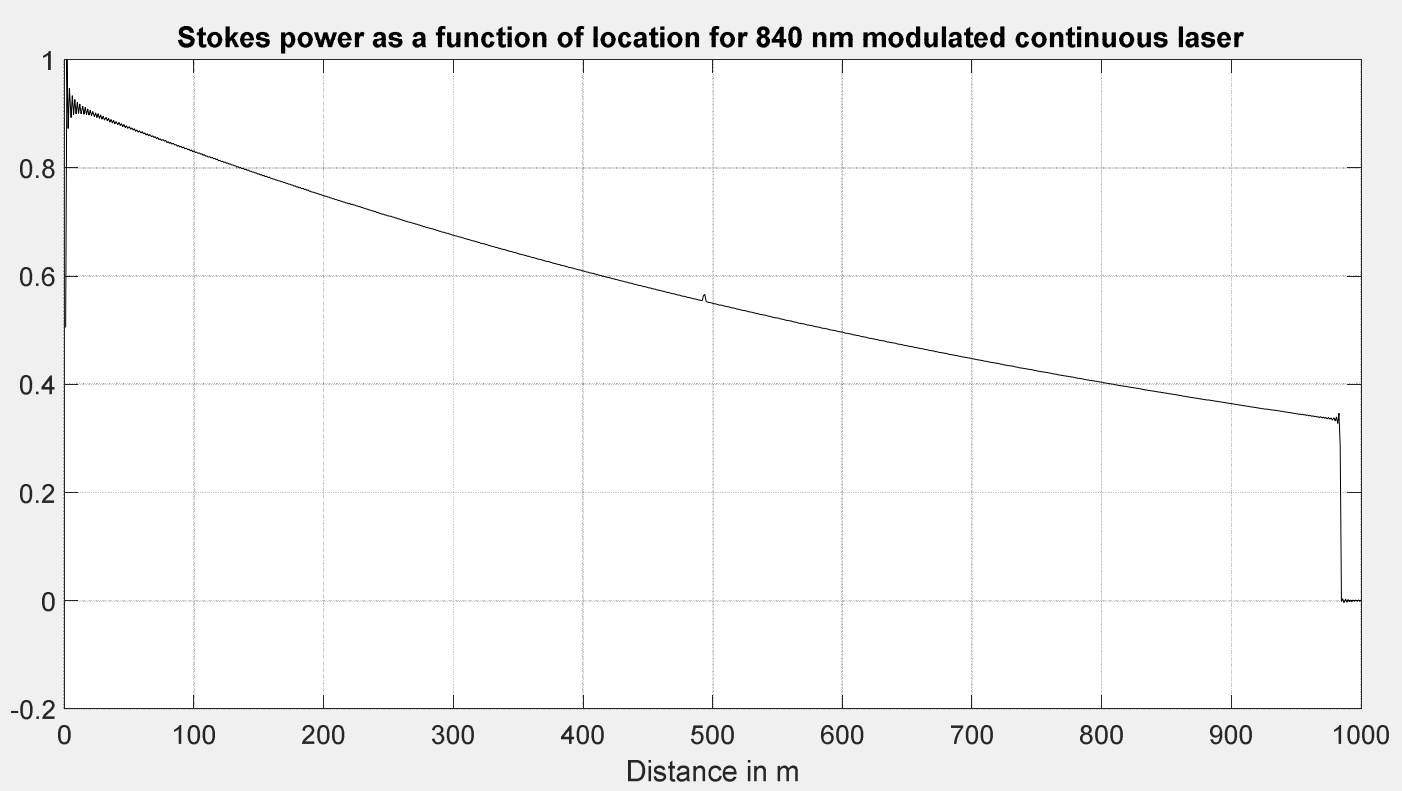

Fig. 3: Stokes power as a function of location of the hot spot for the $840 \mathrm{~nm}$ continuously modulated laser

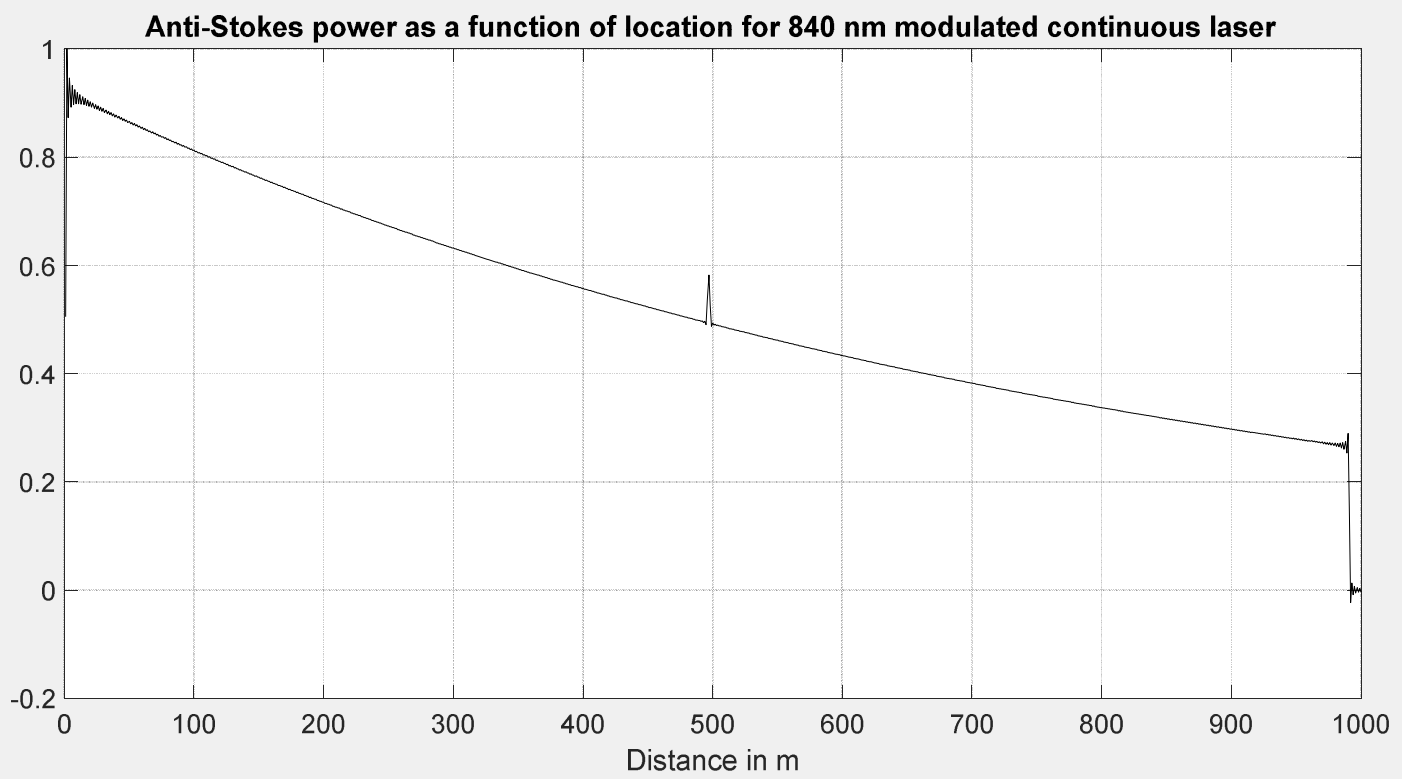

Fig. 4: Anti-Stokes power as a function of location of the hot spot for the $840 \mathrm{~nm}$ continuously modulated laser 


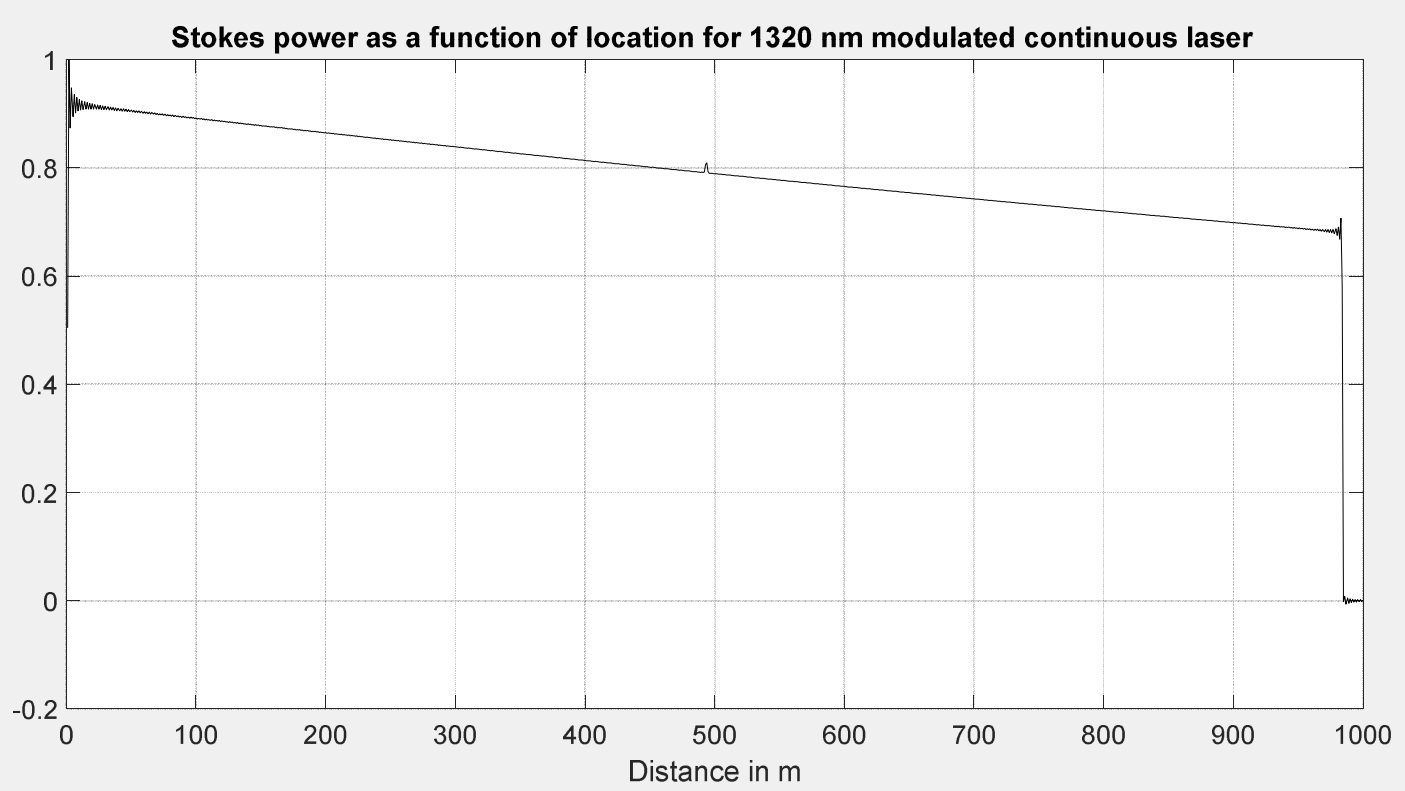

Fig. 5: Stokes power as a function of location of the hot spot for the $1320 \mathrm{~nm}$ continuously modulated laser

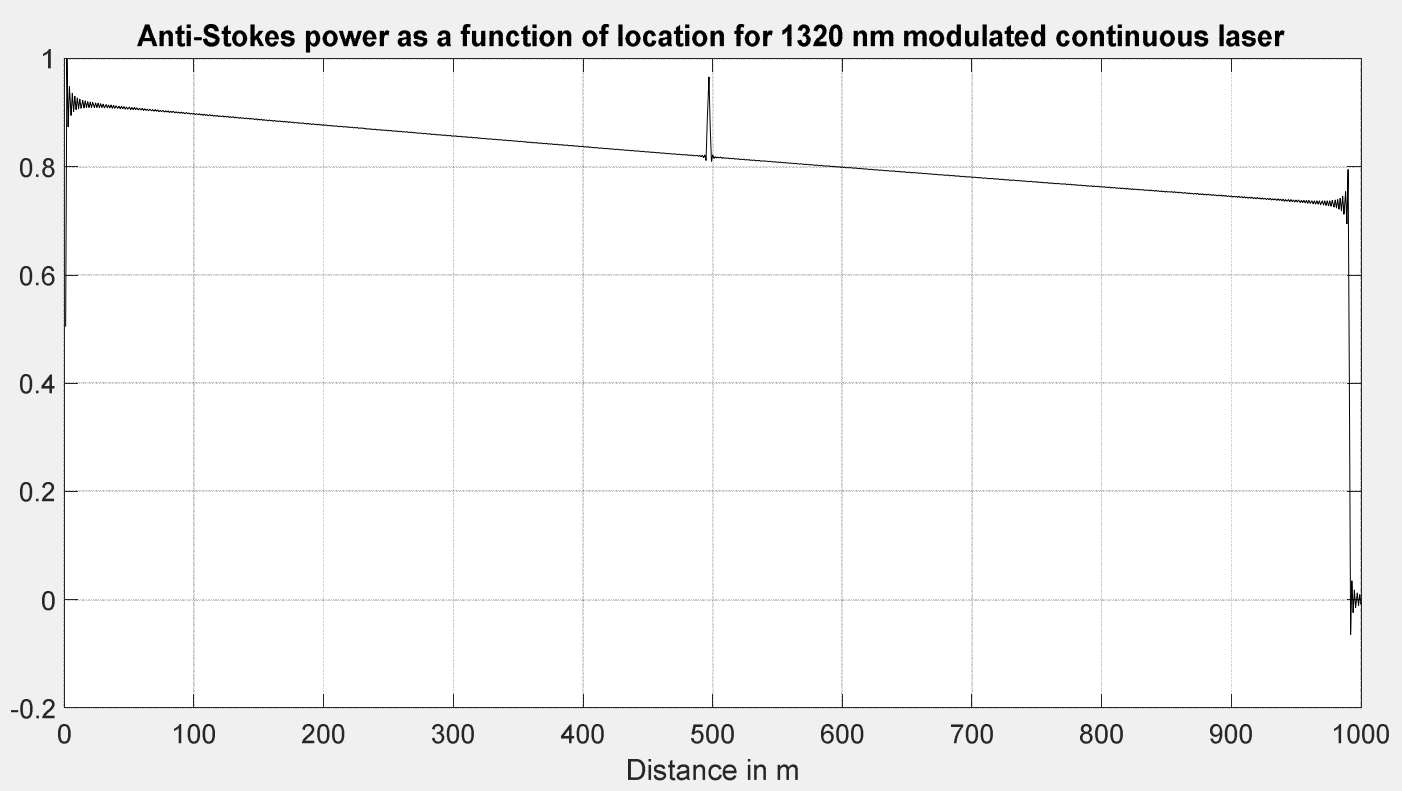

Fig. 6: Anti-Stokes power as a function of location of the hot spot for the $1320 \mathrm{~nm}$ continuously modulated laser 


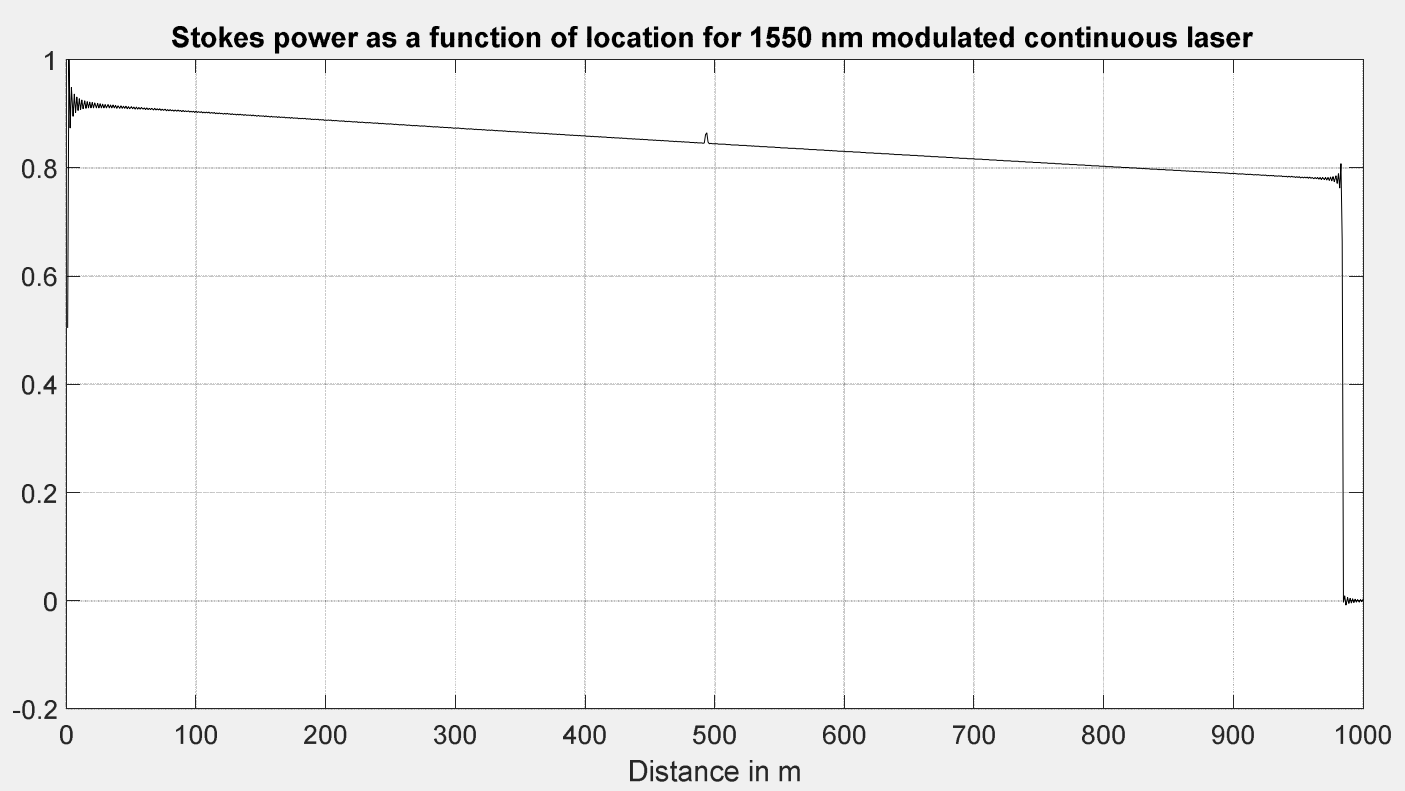

Fig. 7: Stokes power as a function of location of the hot spot for the $1550 \mathrm{~nm}$ continuously modulated laser

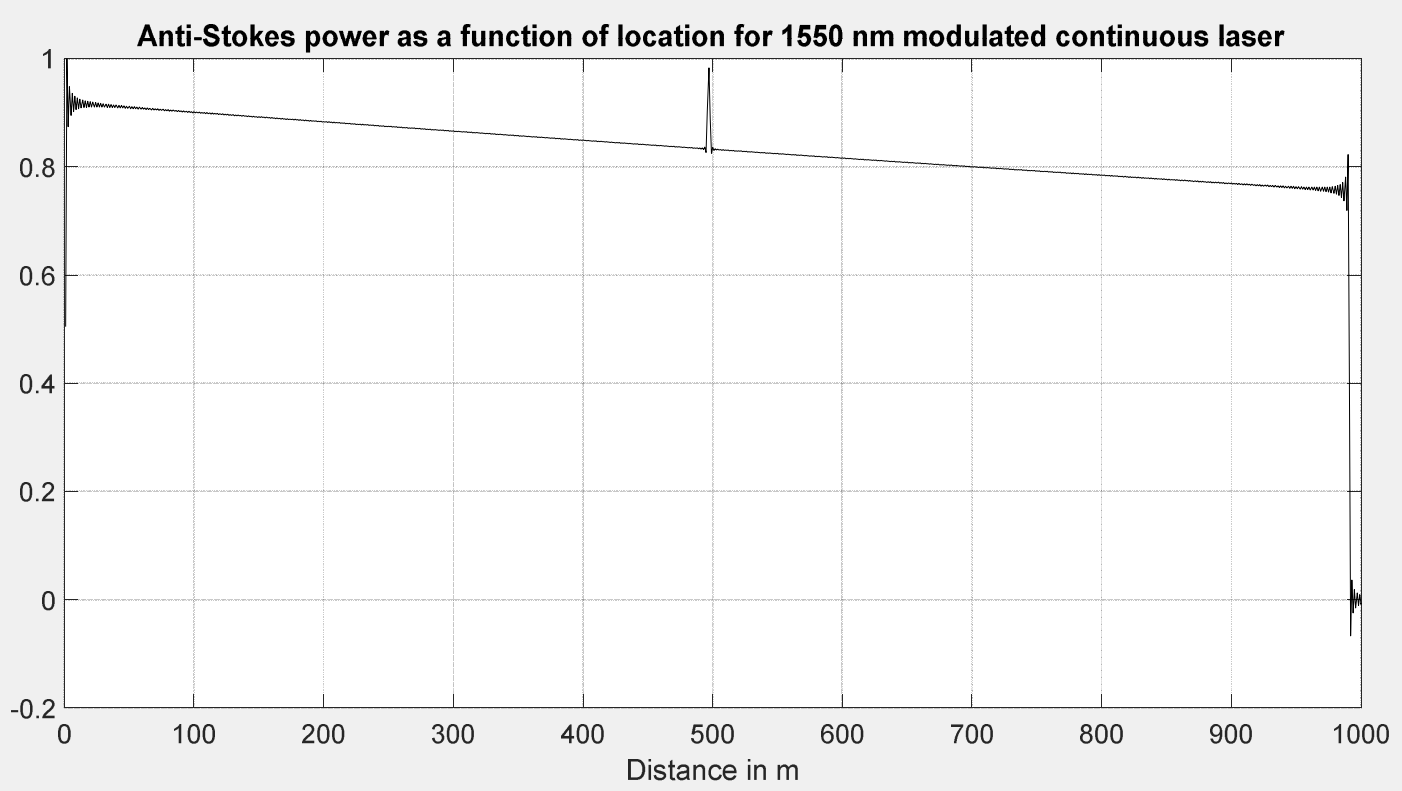

Fig. 8: Anti-Stokes power as a function of location of the hot spot for the $1550 \mathrm{~nm}$ continuously modulated laser 
Modeling results for pulsed laser diodes are presented in figures 9-14. The level of Stokes and anti-Stokes power decreases for increasing wavelength of the laser diodes, and the greater dependence of the Stokes and antiStokes power on to the change of temperature for the higher wavelengths is observed.It is due to the decreasing values of effective power attenuation coefficients and the Raman Stokes and anti-Stokes capture coefficients. Nevertheless, the dependence of the Stokes and anti-Stokes backscattered power on the change of temperature is greater for the higher wavelengths. It is confirmed on the charts by the level of peaks of the backscattered radiation for the distance, where the temperature is increased $\left(47^{\circ} \mathrm{C}\right)$. Also here, the Anti-Stokes lines sensitivity to temperature changes is noticeable.

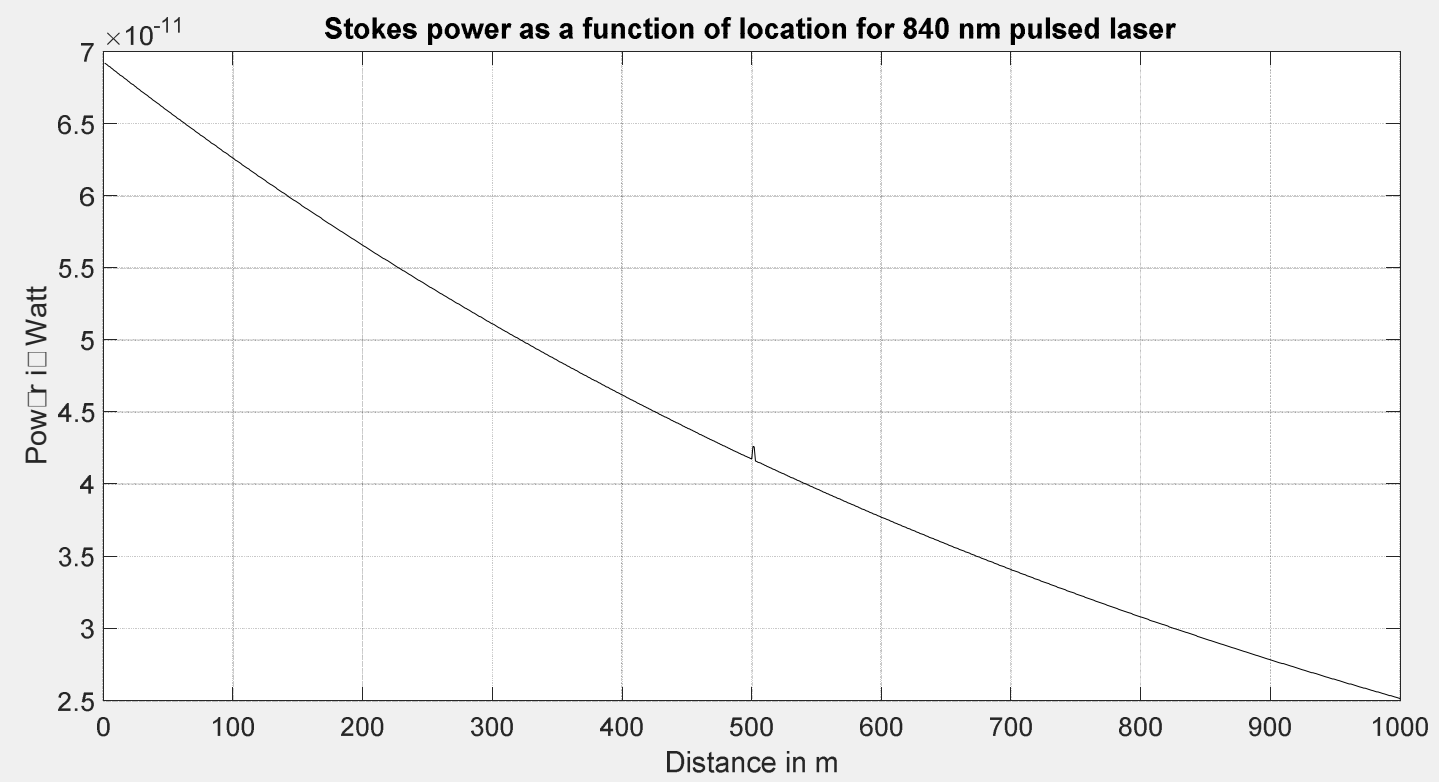

Fig. 9: Stokes power as a function of location of the hot spot for $840 \mathrm{~nm}$ impulse laser (OTDR)

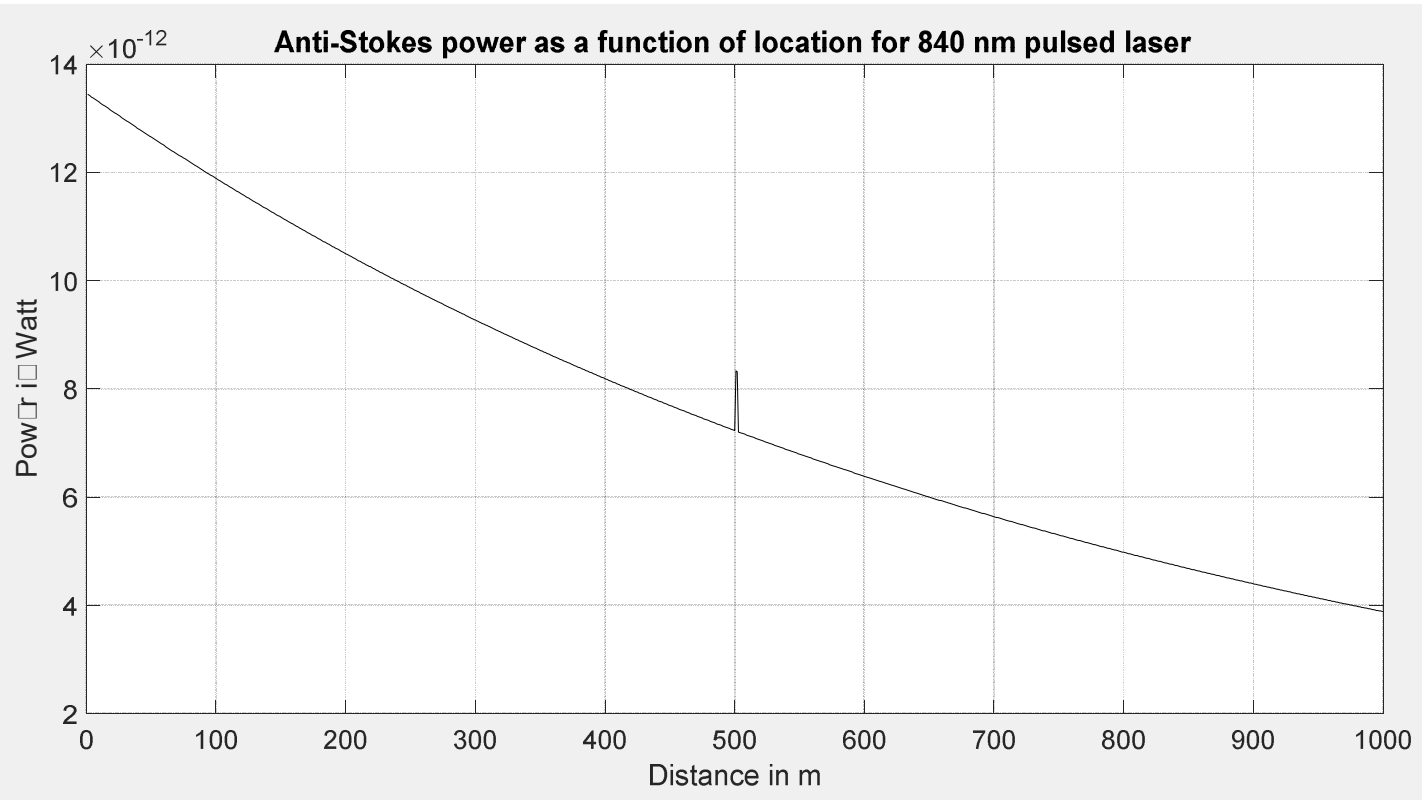

Fig. 10: Anti-Stokes power as a function of location of the hot spot for $840 \mathrm{~nm}$ impulse laser (OTDR) 


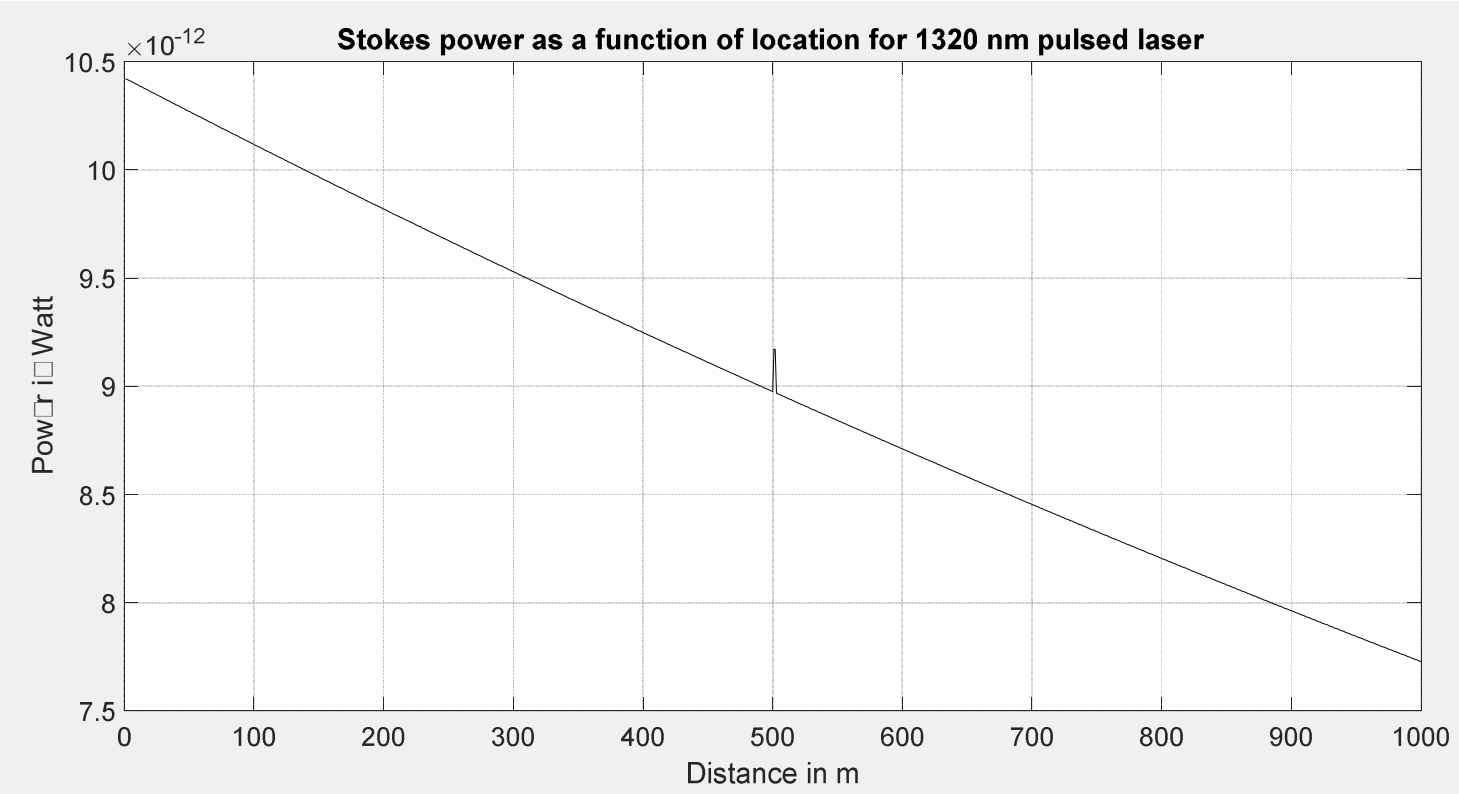

Fig. 11: Stokes power as a function of location of the hot spot for the $1320 \mathrm{~nm}$ impulse laser (OTDR)

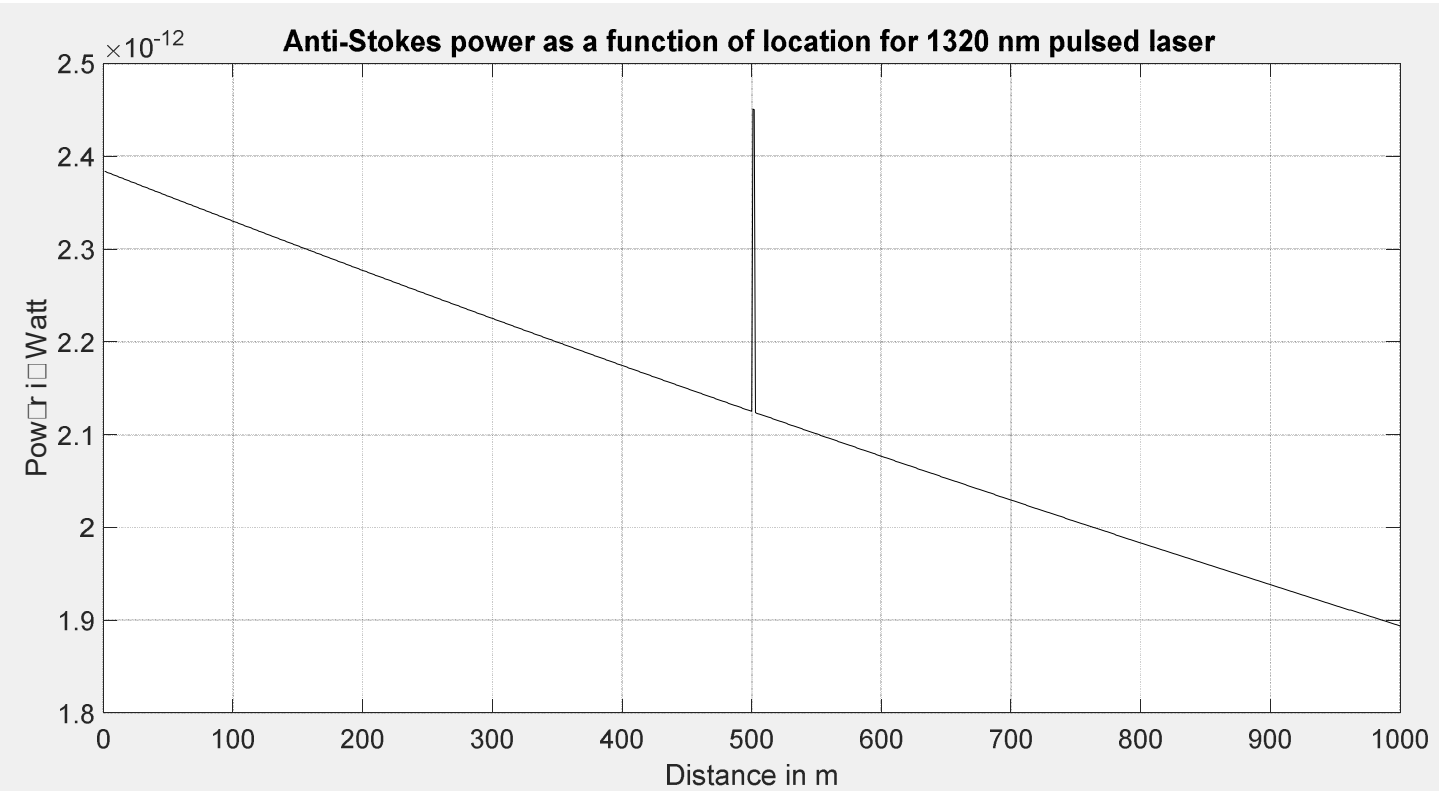

Fig. 12: Anti-Stokes power as a function of location of the hot spot for the $1320 \mathrm{~nm}$ impulse laser (OTDR) 


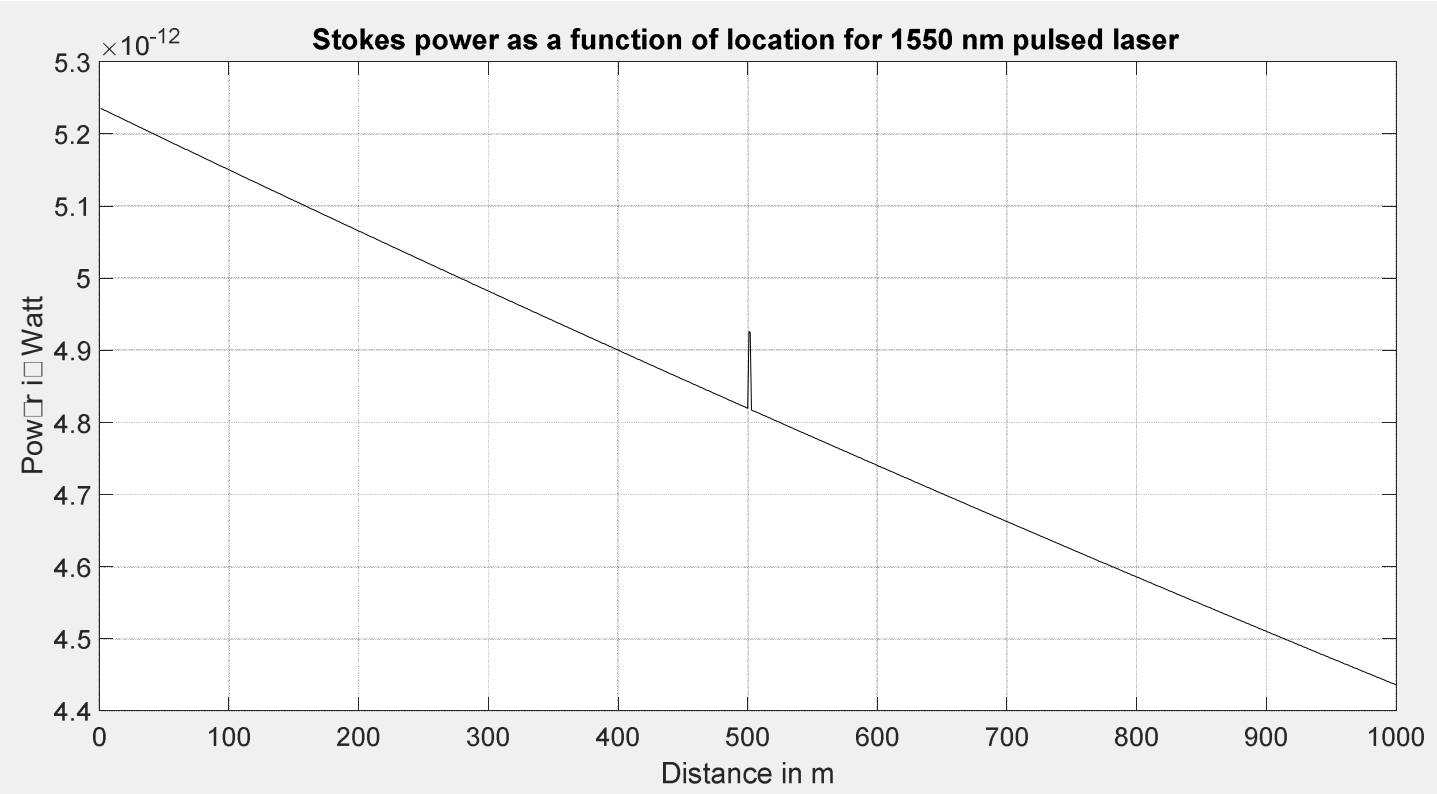

Fig. 13: Stokes power as a function of location of the hot spot for the $1550 \mathrm{~nm}$ impulse laser (OTDR)

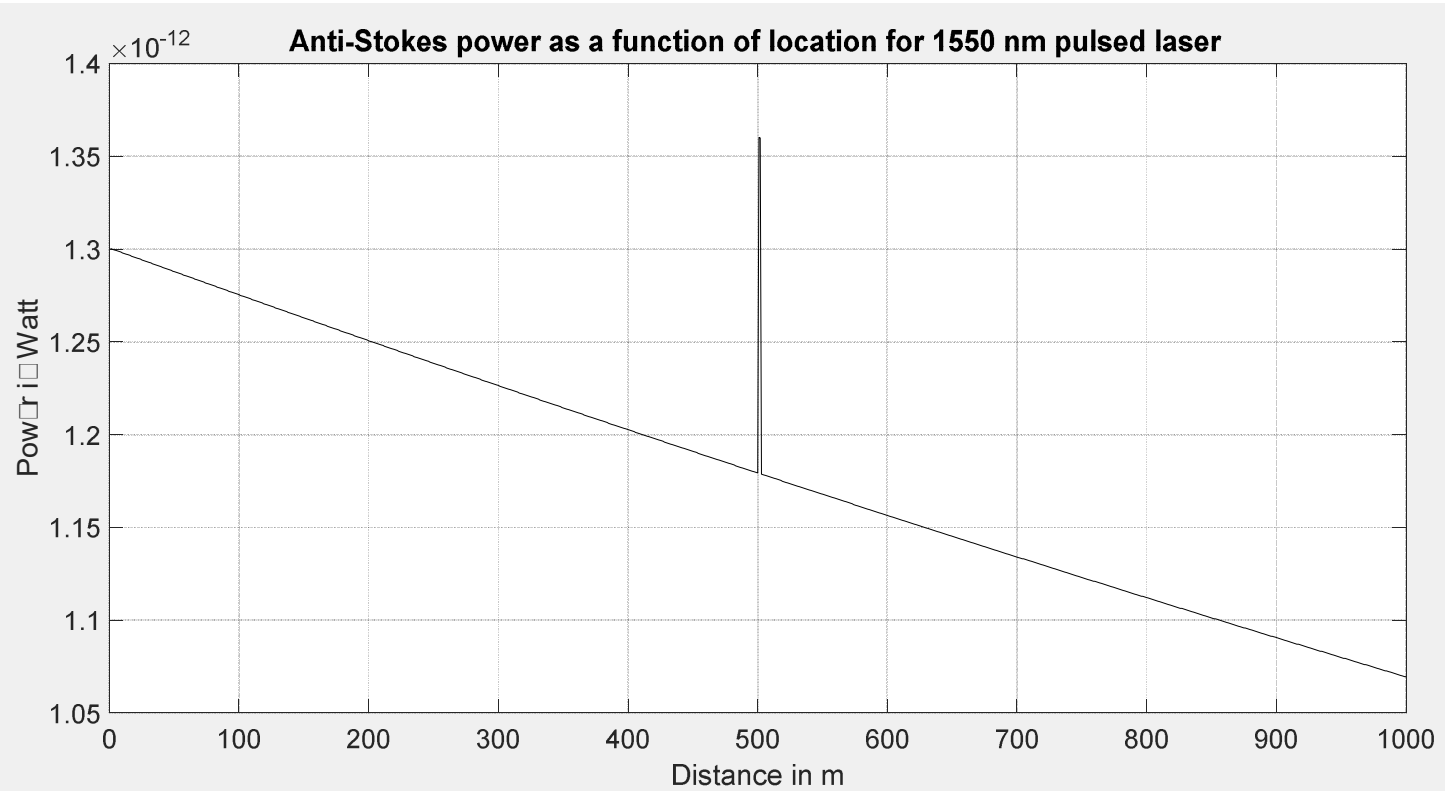

Fig. 14: Anti-Stokes power as a function of location of the hot spot for the $1550 \mathrm{~nm}$ impulse laser (OTDR) 
The ratio between anti-Stokes and Stokes lines is shown on figures 15-17. This ratio is much higher for the higher wavelengths, where the effect of the temperature difference is shown clearly. In the case of $1320 \mathrm{~nm}$ wavelength, the chart is rising because the effective power attenuation coefficient for the Stokes lines is higher than the effective power attenuation coefficient for the anti-Stokes lines.

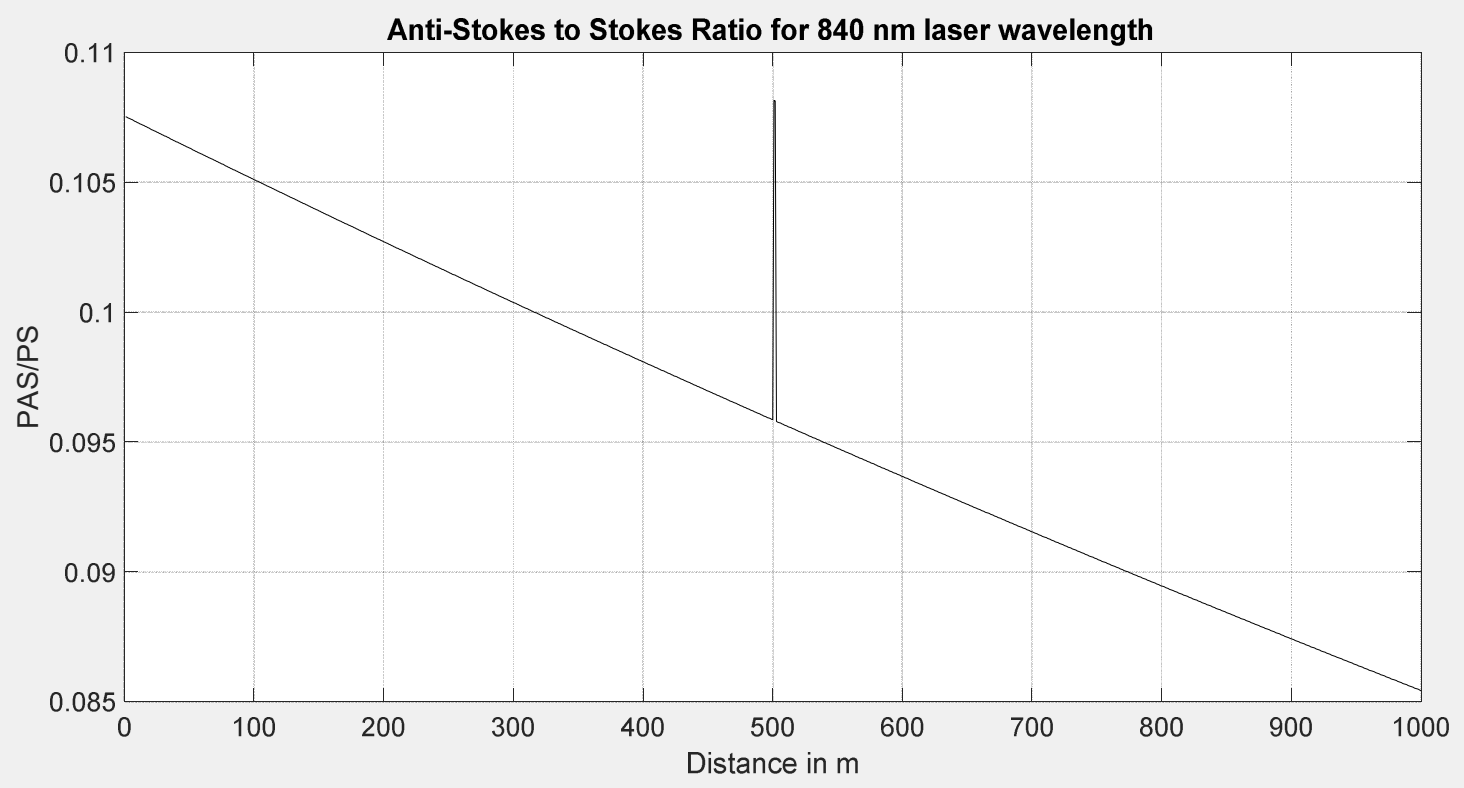

Fig. 15: Anti-Stokes to Stokes ratio for the $840 \mathrm{~nm}$ laser wavelength (OTDR)

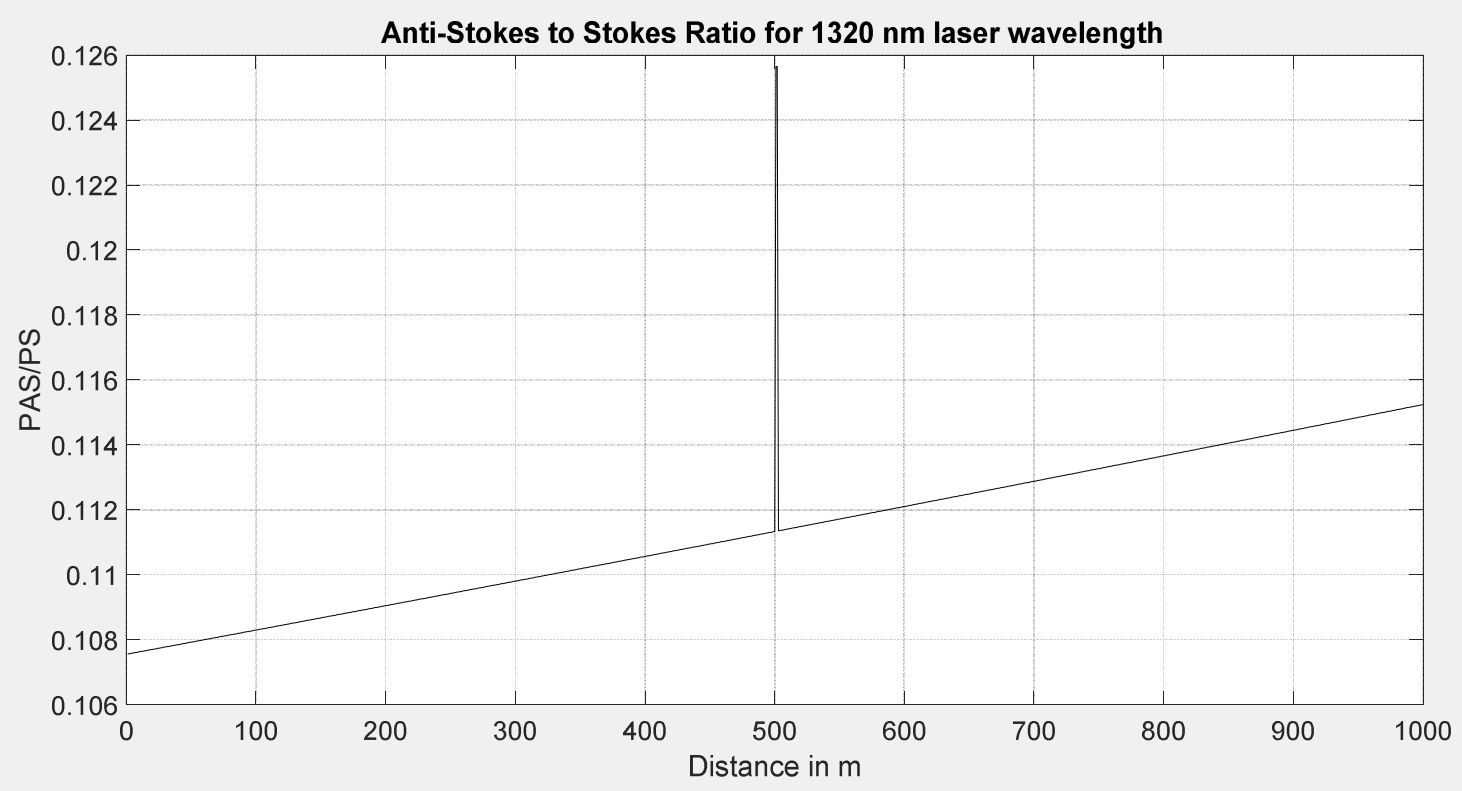

Fig. 16: Anti-Stokes to Stokes ratio for the $1320 \mathrm{~nm}$ laser wavelength (OTDR) 


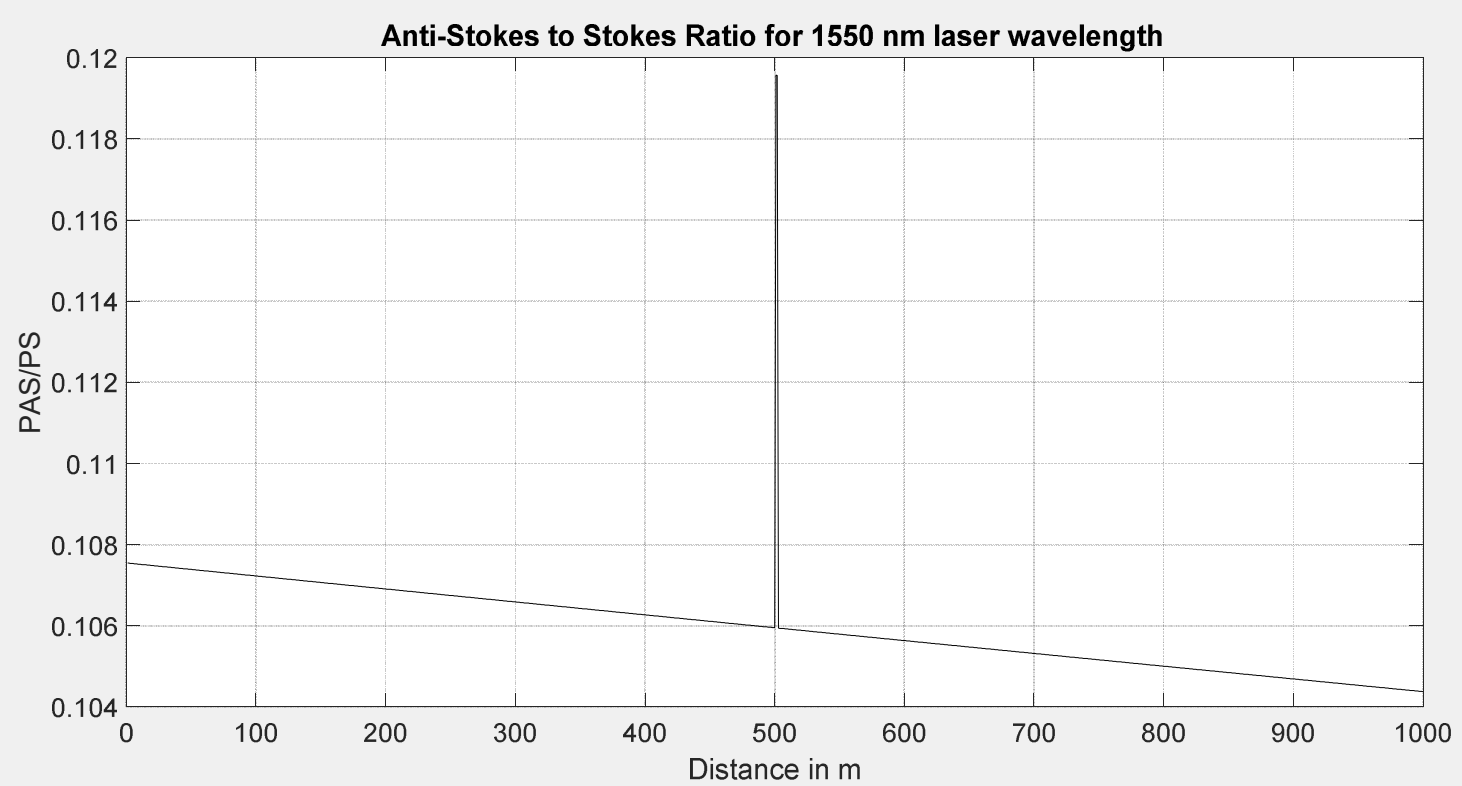

Fig. 17: Anti-Stokes to Stokes ratio for the $1550 \mathrm{~nm}$ laser wavelength (OTDR)

\section{Conclusion}

This paper presented the mathematical models for estimating the expected Raman Stokes and anti-Stokes powers, depending of the wavelength and mode of operation of the laser diode generating the input optical power. The fiber type and length also have an impact on the achieved results. The models confirm the fact that the anti-Stokes lines are more sensitive to temperature changes than the Stokes ones. For both continuous and pulsed lasers, one can notice that the shorter the wavelength, the greater the power of Stokes and anti-Stokes backscattered radiation. In addition, the remark is, that the greater the wavelength of the input radiation, the higher the ratio of anti-Stokes and Stokes backscattered radiation. OTDR is the method that relies on sending single impulse of a laser. It demands a higher optical energy in contrast to OFDR, in which the optical power is spread over the time. The principle of DTS systems based on $O T D R$ is simple and relies on time measurement of the back-scattered signals in order to locate hotspots along a fiber. OFDR is based on more complex analysis of the back-scattered signals and it applies the Inverse Fourier Transformation. Using the results of simulations, a laboratory DTS system is now being built for research purposes. In comparison to the other wavelength of light mentioned in this paper, the chosen laser diode wavelength (1550 $\mathrm{nm}$ ) shows promising results in order to be applied in the research laboratory DTS system.

\section{REFERENCES}

[1] Shatarah I. S. M., Olbrycht R. distributed temperature sensing in optical fibers based on Raman scattering: theory and applications. Measurement Automation Monitoring, vol. 63, no. 02, 2017, pp. 41-44.

[2] Cheng J. X., Xie X. S. Coherent Raman Scattering Microscopy. CRC Press/ Taylor \& Francis Group, 2013, pp. 3-42.

[3] Shatarah I. S. M., Olbrycht R. Distributed temperature sensing in optical fibers based on Raman scattering: demodulation algorythms. Measurement Automation Monitoring, vol. 63, no. 02, 2017, pp. 45-47.

[4] Yilmaz G., Karlik S. E. A distributed optical fiber sensor for temperature detection in power cables. Sensors and Actuators A, vol. 125, 2006, pp. 148-155.

[5] Farahani M. A., Gogolla T. Spontaneous Raman Scattering in Optical Fibers with Modulated Probe Light for Distributed Temperature Raman Remote Sensing. Journal of Lightwave Technology, vol. 17, no. 8, 1999, pp. 13791391.

[6] Bahrampour A.R., Moosavi A., Bahrampour M.J., Safaei L. Spatial resolution enhancement in fiber Raman distributed temperature sensor by employing ForWaRDdeconvolution algorithm. Optical Fiber Technology, 2011, pp. 128-134.

[7] Karamehmedovic E., Jeppesen P., Peucheret C., Bjarklev A. O. Incoherent Optical Frequency Domain Reflectometry for Distributed Thermal Sensing. Technical University of Denmark (2006).

[8] Soto M. A., SignoriniA., Nannipieri T., Faralli S., Bolognini G. High-Performance Raman-Based Distributed FiberOptic Sensing Under a Loop Scheme Using anti-Stokes Light Only. IEEE Photonics Technology Letters,vol. 23 , no. 9, 2011, pp. 534-536. 